BULLETIN Bulletin hispanique

HISPANIQUE Université Michel de Montaigne Bordeaux

123-2 | 2021

Investigaciones semánticas y léxicas actuales

\title{
Mateo Aleman, Guzman de Alfarache, édition et traduction de Monique Michaud
}

Éditions Orbis Tertius, 2021, 2 volumes

Jean-Marc Pelorson

\section{OpenEdition}

Journals

Édition électronique

URL : https://journals.openedition.org/bulletinhispanique/14615

DOI : 10.4000/bulletinhispanique. 14615

ISBN : $1775-3821$

ISSN : $1775-3821$

Éditeur

Presses universitaires de Bordeaux

Édition imprimée

Date de publication : 20 décembre 2021

Pagination : 394-396

ISBN : $979-10-300-0-745-9$

ISSN : 0007-4640

\section{Référence électronique}

Jean-Marc Pelorson, « Mateo Aleman, Guzman de Alfarache, édition et traduction de Monique Michaud », Bulletin hispanique [En ligne], 123-2 | 2021, mis en ligne le 10 décembre 2021, consulté le 07 février 2022. URL : http://journals.openedition.org/bulletinhispanique/14615 ; DOI : https://doi.org/ 10.4000/bulletinhispanique.14615

Ce document a été généré automatiquement le 7 février 2022.

Tous droits réservés 


\section{Mateo Aleman, Guzman de Alfarache, édition et traduction de Monique Michaud}

Éditions Orbis Tertius, 2021, 2 volumes

Jean-Marc Pelorson

\section{RÉFÉRENCE}

Mateo Aleman, Guzman de Alfarache, édition et traduction de Monique MICHAUD, Éditions Orbis Tertius, 2021, 2 volumes en coffret. Vol. $1: 386$ p., Vol. $2: 438$ p. ISBN

978-2-36783-171-8 - ISSN 2265-0776

1 Patine d'ancienneté ou rajeunissement de la langue-cible? Tout traducteur d'ouvrages littéraires du passé est appelé à choisir entre ces deux directions opposées. De la première, le Guzman de Maurice Molho (dans Romans picaresques espagnols, La Pléiade, 1968) reste un exemple retentissant. Il me semble entendre encore (après avoir suivi ses cours de thème en Sorbonne, j'étais devenu à Bordeaux un jeune collègue de Molho) la voix claironnante qui me déclamait à domicile des passages entiers de la vieille version de Chapelain retouchée. Retouches qui ne visaient pas à rapprocher le texte original d'un public français moderne, mais au contraire à remonter vers un âge de notre langue aussi contemporain que possible du castillan de Mateo Aleman. Ce goût élitiste de la traduction archaïsante, invitant le lecteur à savourer en gourmet un âge révolu de deux langues, peut trouver encore aujourd'hui son public, voire des paladins farouches, tel le linguiste Jean-Claude Chevalier. Mais la tendance dominante des traducteurs consiste plutôt désormais à rajeunir en privilégiant de proche en proche les vocables et tournures qui ont le mieux résisté au temps, ou en en empruntant d'autres à un parler récent.

2 C'est bien la solution d'un « rajeunissement discret » ${ }^{1}$ q'a choisie pour sa part Monique Michaud. Mais il faut préciser dans cette orientation une option personnelle : un face-à- 
face permanent et sans intermédiaires avec le texte espagnol, long travail solitaire donc (sur plus de cinquante ans, nous dit-elle) d'un pieux attachement à Aleman ${ }^{2}$. La plupart des autres traducteurs (tels Canavaggio ou Fanlo face à Cervantès) évoquent dans leur paratexte, pour les reprendre ou, plus souvent, les critiquer, les solutions de divers devanciers. Rien de tel chez Monique Michaud. Mais dans cet isolement volontaire nulle trace de narcissisme. Modestement, elle dit craindre que sa traduction «ne grince un peu » par endroits (p. 28), et adopte la position ancillaire de qui vise un idéal de transparence au service d'un « grand prosateur».

Faut-il regretter que la préface ne caractérise pas cette prose, ni le style mis au point pour la rendre en français? Après tout, on n'exige pas d'un musicien interprétant un morceau célèbre qu'il commente la partition et sa manière de jouer. Tout tient à l'exécution et à l'effet produit. Or toute traduction littéraire est aussi une interprétation, que chaque récepteur, avec ce que cela comporte forcément de subjectivité, est appelé à apprécier.

4 Assumant cette subjectivité, je dirai que Monique Michaud a su rendre, d'une façon pour moi inattendue, la verve de Mateo Aleman. On reconnaît vite celui-ci, dans un passage quelconque de son Guzman, de son hagiographie de saint Antoine, ou de son Ortografia castellana, à une profusion de paroles, où se mêlent volubilité andalouse, prédication impétueuse et plaisir de conter à l'adresse d'un lecteur fréquemment apostrophé et bousculé. « C'est parti ! », se dit-on, à la découverte de ces phrases le plus souvent longues et sinueuses, qui nous ballotent en tous sens jusqu'à un terme imprévisible et aussitôt dépassé vers d'autres aventures à la fois affectives et intellectuelles. Pour rapprocher ce texte prolifique d'un public moderne, j'aurais peutêtre choisi, si j'avais envisagé de traduire le Guzman, des solutions proches de celles que j'ai adoptées avec Claude Allaigre pour notre version du Viaje de Turquia ${ }^{3}$ : aération de la mise en page, allégements syntaxiques, bannissement des passés simples au profit de présents de narration ou de passés composés, choix lexicaux poussés vers les usages familiers, voire argotiques, du français d'aujourd'hui. Autres, mais non moins cohérents, ont été les choix de Monique Michaud : tout en respectant les grands blocs de l'original et l'abondance, y compris dans les passages dialogués, des subordonnées, et tout en évitant les néologismes, elle a su rendre le jaillissement et les relances d'un souffle impétueux. Le jaillissement, en conservant par exemple la coordination, souvent rabotée par ses prédécesseurs, du "y » castillan, transposé en " et », clausule de liaison entre événements ou idées (premiers exemples dès le chapitre inaugural, p. 43). La relance, par la pause qu'introduisent, du moins pour qui tente de lire à voix haute, ou en conjuguant image acoustique et déchiffrement, des conjonctions de subordination, telles les nombreux "porque » de l'original, placés en début de phrase ou au cœur d'une longue période. "Car » ou "deux points", dans la traduction, sont des moyens tentants d'allégement, effectivement adoptés par la plupart (Molho compris) des devanciers de Monique Michaud, mais qu'elle a résolument écartés, en faveur de l'effet permanent de justification qui sous-tend surprises de la narration et raisonnements dans la prose alemanienne. Deux exemples entre cent, p. $64:$ : Parce que dire qu'il existe des philtres ou des poudres pour aimer, c'est faux », puis :

L'amour doit être libre. Il doit faire l'abandon des facultés de l'âme à l'objet aimé ; parce que le gouverneur de la forteresse ne livre pas la place quand on la lui prend de force, de même que celui qui aimerait par des maléfices, on ne peut pas dire qu'il aime, parce qu'il va contraint et forcé là où ne le conduit pas sa libre volonté. 

des pauses rythmiques qu'on apprend à apprécier quand on les oralise, de même qu'on peut s'habituer au gueuloir de Flaubert ou au verset claudélien. Très audible donc pour «l'oreille interne », la traduction de Monique Michaud.

D'autant que l'oralité y est rendue non seulement dans les passages dialogués, mais, plus subtilement, dans les monologues de l'instance narratrice ou des personnages. D'une grande justesse phonique, tel un coup d'archet à rebours, résonne l'incise (p. 48) : " en castillan, je veux dire ", avec son refus d'inversion, ou encore cette note (p. 165) au bas de l'histoire d'Ozmin et Daraja: «Emporté par le récit, Aleman passe ici à la seconde personne du singulier, substituant au conteur (le prêtre s'adressant à ses compagnons de route) le narrateur (Guzman s'adressant à son lecteur). » La succession des voix, les surprises et bousculades de leur polyphonie, typiques de l'écriture alémanienne, sont ainsi bien rendues et bien commentées.

Dans une solide préface qui replace sobrement l'ouvrage en son temps, attachante est l'image que propose la traductrice d'un Mateo Alemán meurtri par la vie et mal consolé de ses déboires par le fulgurant succès de son Guzman. Quant à savoir si l'idéologie sousjacente à ce roman relève d'une orthodoxie chrétienne strictement conforme à la discipline tridentine et confirmée par le San Antonio de Padua, il serait trop long et trop étranger à mes compétences d'en discuter ici. Disons seulement que Monique Michaud, dans une étude remarquable dont sa préface reprend l'essentiel ${ }^{4}$, a su mettre en valeur le sens de l'humain montré par Aleman quand il a affronté, en tant que juge enquêteur, vainement mais courageusement, le « mur de la finance » représenté à l'époque par les Fugger et leur féroce exploitation des forçats travaillant aux mines d'Almadén. Comment rompre le cycle infernal délinquance-répression, la question, à laquelle Cervantès s'est aussi attaqué, reste actuelle dans sa complexité. Eclipsé par le Quichotte, qui lui doit beaucoup, le Guzman n'est pas devenu pour autant un objet de musée. Et ne se réduit pas à une sorte de catéchisme tridentin, vue simpliste qui caricaturerait les prises de position de Monique Michaud: elle n'aurait pas consacré tant d'années à sa patiente méditation si elle n'avait été sensible à l'audace novatrice d'un «conteur accompli ", co-fondateur avec Cervantès du roman moderne en tant qu'aventure fictive où se politise la satire d'une société qui va de travers. Relancée et rajeunie par la traduction, la lucidité mordante d'un galérien repenti est faite pour nous atteindre encore, comme continue à nous interpeller la quête maladroite et inaboutie d'un élan chevaleresque obstiné.

\section{NOTES}

1. Cette formule, que j'ai proposée dans la préface du Guide de la version Classique chez Armand Colin en 1972, a été reprise en 2001 par Jean Canavaggio dans sa présentation des CEuvres romanesques complètes de Cervantès, Gallimard, La Pléiade, 2001, t. 1, p. LXXIV.

2. Le silence de Monique Michaud sur ses devanciers n'interdit pas des comparaisons, notamment avec l'édition du Guzman publiée en 2014 par Francis Desvois, dans les Classiques 
Garnier. Mais ce serait un travail de longue haleine qui dépasse les possibilités du présent compte rendu.

3. Voyage en Turquie. Dialogue entre Pierre de Méchantour, Jean d'Escroquendieu et Dédé Couandouce, Paris, Éditions Bouchène, 2013.

4. Almadén. Le commissaire Aleman mène l'enquête, Les Cahiers Forell, $\mathrm{n}^{\circ}$ 7, p. 133-185, Poitiers, 1996.

\section{AUTEURS}

\section{JEAN-MARC PELORSON}

Université de Poitiers 